\title{
Chamber material effects on actinometric measurements in rf glow discharges
}

\author{
Tina J. Cotler \\ IBM East Fishkill, Hopewell Junction, New York 12550
}

Michael L. Passow

Department of Nuclear Engineering University of Michigan, Ann Arbor, Michigan 48109

Jeffrey P. Fournier

Solid State Electronics Laboratory Department of Electrical Engineering and Computer Science, University of Michigan, Ann Arbor, Michigan 48109-2122

Mary L. Brake

Department of Nuclear Engineering University of Michigan, Ann Arbor, Michigan 48109

Michael E. Elta

Solid State Electronics Laboratory Department of Electrical Engineering and Computer Science University of Michigan, Ann Arbor, Michigan 48109-2122

(Received 4 June 1990; accepted for publication 26 November 1990)

\begin{abstract}
The relative concentration of atomic fluroine was measured in $\mathrm{CF}_{4} \mathrm{rf}$ glow discharge using the actinometric technique. The dependence of fluorine concentration on power, pressure and flow are presented and shown to be dependent upon reactor wall material and electrode material.
\end{abstract}

\section{INTRODUCTION}

The technique of inert gas actinometry was first introduced to study $\mathrm{CF}_{4}$ and $\mathrm{CF}_{4} / \mathrm{O}_{2}$ rf glow discharges. ${ }^{1,2}$ It has been used to study numerous other fluorinated gas systems since then. ${ }^{3-6}$ In addition, actinometry has been compared with numerical models for model verification. ${ }^{7,8}$ The systems utilized in the above references were constructed with a variety of materials (e.g. alumina, stainless steel). This study reports the effect of reactor material on the atomic fluorine concentration as measured using the actinometric technique. First the reactor used in this work will be described. Next, the actinometric technique will be briefly outlined ( the above references contain a more complete description). Finally, the trends in fluorine concentration versus power, pressure, and flow for various electrode and reactor wall materials will be presented and discussed. Comparisons between the trend observed here and those predicted by numerical models ${ }^{9,10}$ and other experimental measurements ${ }^{11}$ will be provided. However, bear in mind that the mass spectrometry measurements in Ref. 11 are made downstream and there is uncertainty in some rates used in current chemical kinetics models. In addition, the residence times typical in our commercial reactor are significantly longer than those treated in the above references. Therefore the agreement, or disagreement, may be coincidental in some instances.

\section{EXPERIMENT}

This study was carried out in a commercial parallel plate radial flow reactor (SEMI Group $1000 \mathrm{TP} / \mathrm{CC}$ ) with etch gas entering through a showerhead electrode at the top of the 43-cm-diam chamber and exiting through a 10-cm exit port directly below the lower electrode. The etch tool and pertinent ancillary equipment are shown in Fig. 1. The vari- able plate spacing was set to $6 \mathrm{~cm}$ for this work. The upper grounded electrode was $40 \mathrm{~cm}$ in diam and made of anodized aluminum and the lower powered electrode was $30 \mathrm{~cm}$ in diam and made of quartz-covered (4.5-mm-thick) alumi-

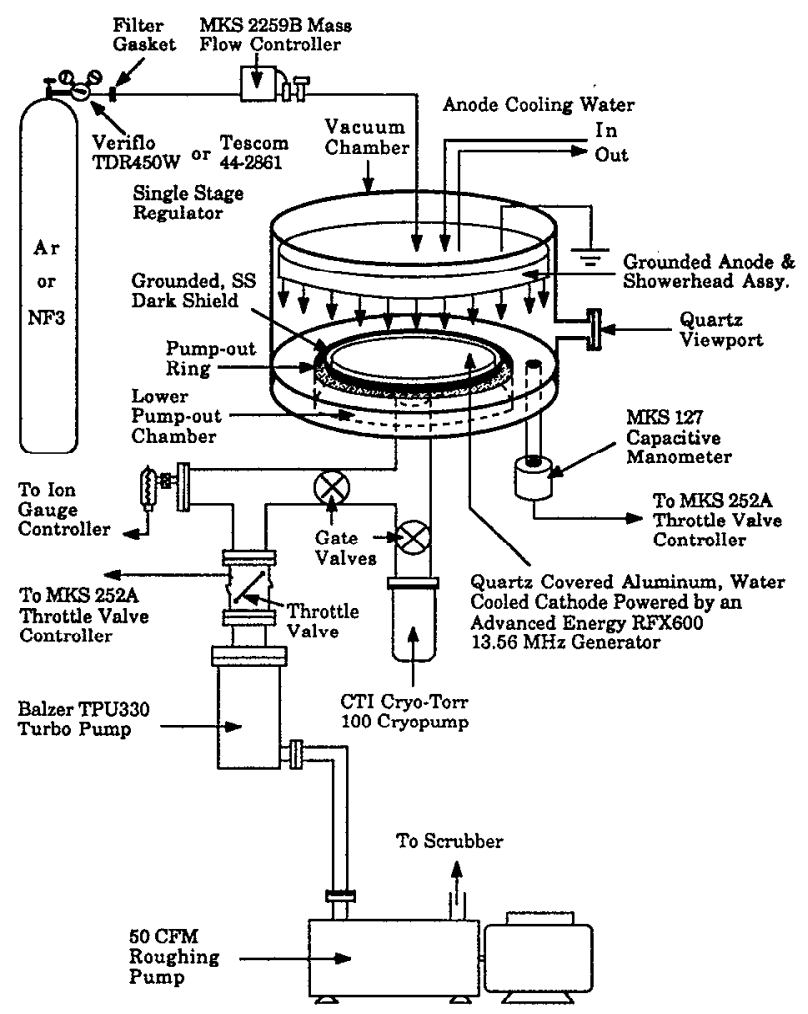

FIG. 1. RIE tool and ancillary equipment. 
num. The walls were covered with flame spray coated aluminum oxide. Power was provided by an Advanced Energy RFX600 $13.56 \mathrm{MHz}$ RF generator. A stainless steel dark shield surrounded the lower electrode $(4 \mathrm{~mm}$ from the electrode) to confine the plasma. The gas inflow was controlled by a MKS 2259B (0-100 sccm and 0-10 sccm nitrogen equivalent for $\mathrm{CF}_{4}$ and argon, respectively) mass flow controller ( $\mathrm{mfc}$ ) and the outflow was regulated with a MKS $252 \mathrm{~A}$ automatic throttle valve controller. The sensor for the controller was a MKS 127 capacitive manometer. The reactor chamber was pumped with a Balzer TPU330 turbomolecular pump and backed by a mechanical pump. In addition, the chamber was connected to a CTI-Cryo-Torr 100 cryopump which was used to obtain a repeatable base pressure. A Techware PAL 68000 (a generic equipment controller) was used to control the etch tool.

In addition to the reactor described above, two inserts were made of stainless steel and aluminum. The inserts fit inside the chamber and completely covered the aluminum oxide walls exposed to the plasma. Blanks, made of the same materials as the inserts, were included to cover the quartz cathode. The blanks were electrically isolated from the cathode by the quartz plate. The automatic matching network tunes the coupling capacitance to account for any additional series capacitance due to the metal blank. This did not appear to change the electrical properties of the discharge appreciably since the $\mathrm{dc}$ bias remained approximately the same when the blanks were used to cover the quartz electrode. The major effect of these blanks on the electrical properties of the discharge is the difference in the secondary electron emission coefficients of the blanks versus the quartz. $\mathrm{A} \mathrm{CF}_{4}$ plasma was run for $4 \mathrm{~h}$ after insertion of new wall and/or cathode materials to passivate the inserts.

Plasma-induced emission was monitored using an EG\&G PARC 1460 optical multichannel analyzer, with an EG\&G PARC 1421 intensified 1024 channel photodiode array coupled to a Jarrell Ash $0.25-\mathrm{m}$ spectrograph with 3 interchangeable gratings $(150,600,1200$ groove $/ \mathrm{mm})$. The entrance slit was illuminated via a fiberoptic cable connected to a quartz window located $4.1 \mathrm{~cm}$ above the lower electrode.

The actinometric technique allows the relative concentration of a ground-state atomic species to be determined under certain conditions. This is achieved by introducing a small amount of an inert gas as an actinometer to which the atomic species can be referenced. The specific requirements for validity of this technique are: (1) the emission from two species must arise from de-excitation of higher level states which are similar in energy, (2) the higher energy states are created by electron impact excitation of atoms in the ground state, and (3) the electron impact excitation cross sections are similar in shape and threshold energy. In this investigation, the actinometer used is argon and the atomic species being measured is fluorine. The relative concentration of the species of interest is given by

$$
\frac{n_{\mathrm{F}}}{n_{\mathrm{Ar}}}=K \frac{I_{\mathrm{F}}}{I_{\mathrm{Ar}}},
$$

where $n_{\mathrm{F}}$ and $n_{\mathrm{Ar}}$ are the respective fluorine and argon concentrations, $I_{\mathrm{F}}$ and $I_{\mathrm{Ar}}$ are the respective fluorine and argon emission intensities, and $K$ is an undetermined parameter which is approximately constant under conditions where the actinometric technique is valid. In this experiment the 704$\mathrm{nm}$ fluorine line together with the 751-nm argon linc were used. This allows the trends in fluorine atom concentration to be determined versus process conditions when the above criteria have been met. The measurement technique has been shown to be valid for estimating fluorine concentrations by examining the spectral line shape. ${ }^{5}$ However, this technique does not allow absolute concentrations to be measured due to the presence of the unknown parameter $K$.

The conditions used in this study are: gas flow from 10 to $40 \mathrm{sccm}$ and pressure from 50 to $300 \mathrm{mT}$. This results in residence times from approximately $500 \mathrm{~ms}$ to $12 \mathrm{~s}$. The inert gas used as the actinometer was argon as discussed in the previous references. The argon flow was set to $5 \%$ of the $\mathrm{CF}_{4}$ flow for all experiments.

\section{RESULTS AND DISCUSSION}

Figures 2-5 show the ratio of the emission intensity of fluorine to argon versus gas flow for the various material combinations for two pressures, 300 mTorr (Figs. 2-4) and $50 \mathrm{mTorr}$ (Fig. 5), and for several power settings. This ratio is proportional to the relative concentration of fluorine as described above. The five combinations of reactor materials are: (a) reactor as described above (alumina walls, quartz electrode), (b) stainless-steel walls, quartz electrode, (c) aluminum walls, quartz electrode, (d) stainless-steel walls and electrode, and (e) aluminum walls and electrode. As expected, for all materials and all flow settings, the atomic fluorine concentration increased as power increased. However, there was significant variation in the relative fluorine

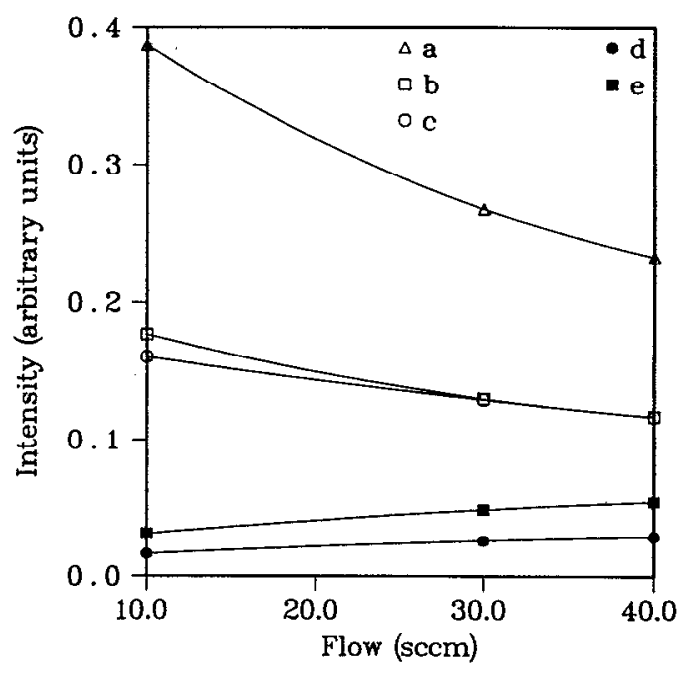

FIG. 2. Ratio of the emission intensity of fluorine to argon vs gas flow at 300 $\mathrm{mTorr}, 350 \mathrm{~W}$ for different reactor materials. (a) alumina walls, quartz electrode, (b) stainless steel walls, quartz electrode, (c) aluminum walls, quartz electrode, (d) stainless steel walls and electrode, (e) aluminum walls and electrode. 


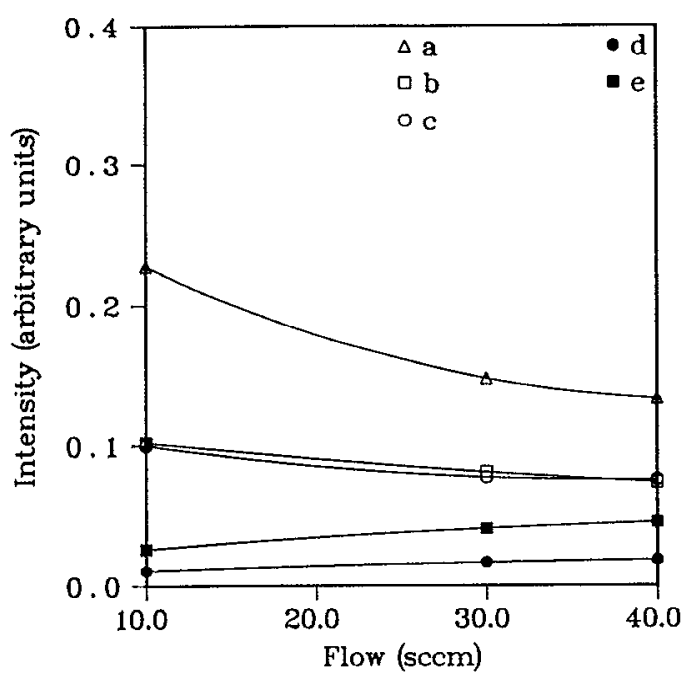

FIG. 3. Ratio of the emission intensity of fluorine to argon vs gas flow at 300 mTorr, $250 \mathrm{~W}$ for different reactor materials. Reactor material combinations for (a)-(e) detailed in Fig. 1.

concentration with respect to the different reactor materials. As can be seen in Figs. 2-5, for all process conditions, the quartz and alumina combination gave the highest fluorine concentration, the metal walls with quartz electrode gave a somewhat lower fluorine concentration, and the metal walls and electrodes gave the lowest fluorine concentration. Even though the etching reaction which occurs at the quartz electrode suppresses the fluorine concentration, this is overshadowed by the fact that oxygen is also produced when the quartz is etched. Small amounts of oxygen liberated in the etching reaction significantly enhance the amount of free fluorine by competing with the recombination reaction: ${ }^{9}$

$$
\mathrm{CF}_{3}+\mathrm{F}_{\rightarrow}^{M} \mathrm{CF}_{4}
$$

via the reaction: ${ }^{12}$

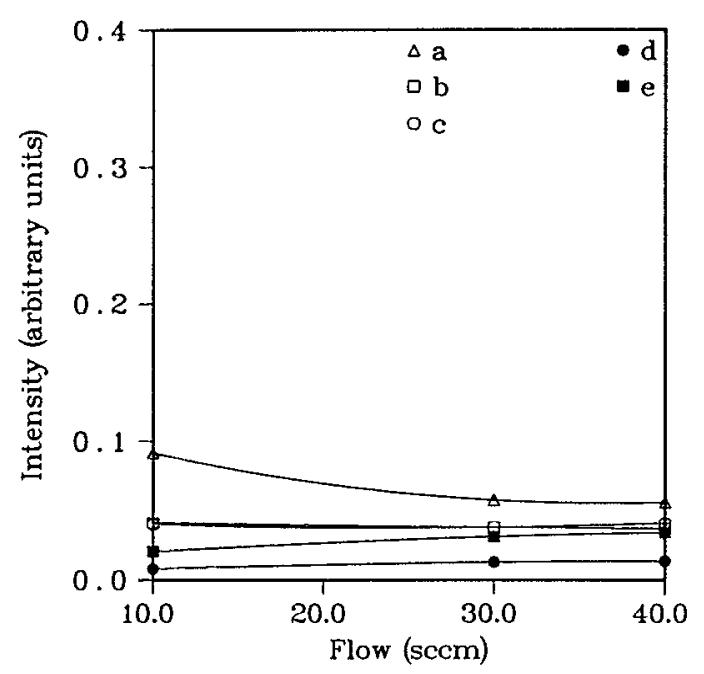

FIG. 4. Ratio of the emission intensity of fluorine to argon vs gas flow at 300 mTorr, $150 \mathrm{~W}$ for different reactor materials. Reactor material combinations for (a)-(e) detailed in Fig. 1.

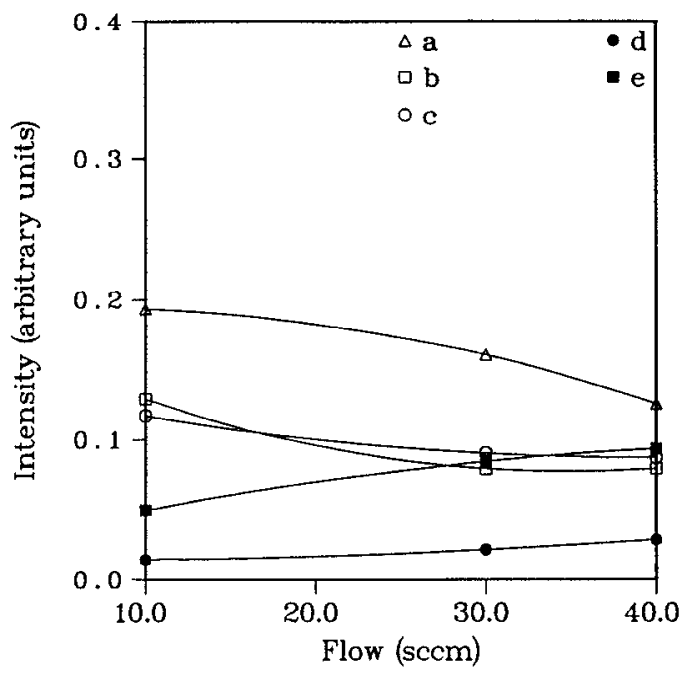

FIG. 5. Ratio of the emission intensity of fluorine to argon vs gas flow at 50 mTorr, $250 \mathrm{~W}$ for different reactor materials. Reactor material combinations for (a)-(e) detailed in Fig. 1.

$$
\mathrm{CF}_{3}+\mathrm{O} \rightarrow \mathrm{COF}_{2}+\mathrm{F} \text {. }
$$

It is well known that alumina retains large amounts of water which can also be a major source of oxygen, particularly during discharge conditions. Thus the maximum amount of oxygen is produced with alumina walls and a quartz electrode, enhancing the fluorine concentration by inhibiting reaction (2) (the recombination reaction). Note that the effect of the aluminum and the stainless-steel walls on the fluorine concentration appears to be quite similar. In other words, the difference in the effect of the aluminum and stainless-steel materials on the atomic fluorine concentration is small.

To determine the effect of the addition of trace amounts of oxygen on the fluorine concentration, a small amount of $\mathrm{O}_{2}$ (less than $0.25 \%$ of the $\mathrm{CF}_{4}$ flow) was intentionally introduced into the discharge for the case (case $e$, all aluminum chamber) where there was no internal source of oxygen (neglecting small amounts of oxygen liberated by desorption which are present regardless of the external source). The percent of oxygen in the mixture is stated as less than $0.25 \%$ since the mass flow controller readout is accurate only to 0.1 sccm, and the flow valve was throttled back so that the flow was less than $0.1 \mathrm{sccm}$. The fluorine concentration at 30 sccm in Fig. 3(c) (aluminum walls with quartz electrode) versus Fig. 3(e) (all aluminum chamber) is $(0.076 / 0.046=1.66)$ approximately double. The intentional addition of oxygen to an all aluminum chamber (when compared to aluminum walls with the quartz electrode) yields a similar increase in fluorine concentration of $(0.075 / 0.041=1.83)$, also approximately double. Large increases in fluorine concentration with oxygen addition have been predicted by computational models ${ }^{9,10}$ and observed by mass spectrometry measurements in Ref. 11.

The liberation of oxygen from chamber materials is expected to be strongly dependent on the electron energy distribution function and the ion density and energy (which were not measured for these experiments). This is supported 
by the fact that at low power (Fig. 4) there was not much difference between the quartz electrode and metal electrode cases whereas at high power the difference is quite large. Less removal of the oxygen containing material and thus less oxygen liberation appears to occur, presumably due to lower electron energies and densities (compared to higher powers) as well as lower ion density and less energetic bombardment (lower dc bias). The fluorine concentration remains approximately the same for the three power levels for metal electrodes and walls, whereas it increases dramatically with increased power for the quart $\angle$ electrode and metal or alumina walls. The highly nonlinear dependence of fluorine concentration on power suggests oxygen liberation increases with power (and hence ion energy) for the cases with an oxygen containing electrode only. The fluorine concentration was observed to decrease with increasing pressure in Ref. 11 . However, the two computational models disagree: Ref. 10 fails to predict this trend while Ref. 9 does.

The variation of fluorine concentration with flow rate was also examined. The fluorine concentration was expected to decrease with increasing flow, since the residence time is decreased. This trend has been observed by mass spectrometry measurements. ${ }^{11}$ However, the two computational models disagree: Ref. 10 predicts this trend while Ref. 9 predicts a minimal change in fluorine concentration with flow. As seen in Figs. 2-4, this trend is observed here; the fluorine concentration decreased with increasing flow for the cases which contained the quartz electrode. The opposite trend is seen where the reactor walls and electrode are completely covered with aluminum and stainless steel. These materials cause the fluorine concentration to be decreased to a level sufficient to reverse the trend. Flamm noted this trend for a reaction with silicon although he did not study the material effects. ${ }^{13}$ In his experiment, the silicon was said to "load" the free fluorine. Specifically, by utilizing the available fluorine in an etch reaction, the conversion was increased by

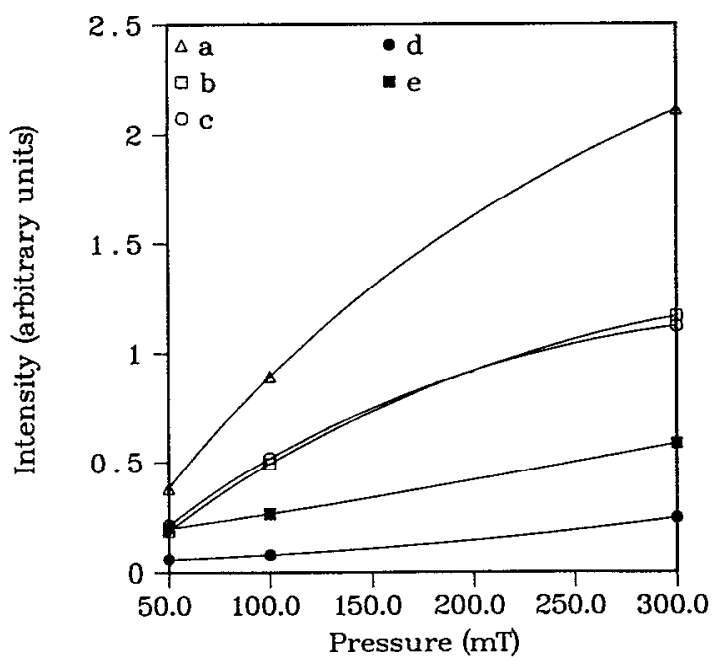

FIG. 6. Ratio of the emission intensity of fluorine to argon vs pressure for different reactor materials at $30 \mathrm{sccm}$ and $250 \mathrm{~W}$. Reactor material combinations for (a)-(e) detailed in Fig. 1. blocking the recombination reaction (reaction 2). In the case of aluminum and stainless-steel materials investigated here, the utilization of available fluorine can be explained in two ways. First, the involatile fluorinated layer that forms may be sputtered off permitting the use of fluorine. However, the ion energies are fairly low under these conditions $(\approx 150-350 \mathrm{~V})$. Second, these materials may promote recombination of fluorine, for example to $F_{2}$. The fact that the presence of stainless steel reduces the fluorine concentration was observed in Ref. 11. Although they suggest the stainless steel reacts with fluorine, the mechanism was not specified.

Figure 6 shows the relative concentration of fluorine versus pressure at $30 \mathrm{sccm}$ and $250 \mathrm{~W}$. The argon emission data was divided by the argon pressure to provide the argon emission at constant argon density. ${ }^{3}$ The fluorine concentration is expected to increase with pressure from a residence time argument. This trend is indeed seen for all material combinations in Fig. 6.

\section{SUMMARY AND CONCLUSION}

It has been shown that chamber material effects play an important role in the net amount of fluorine generated in an rf glow discharge. The effect of reactor materials is most important when attempting to compare, for example, computed values to concentration trends measured by actinometry or the results from two different experimental setups. Thus, material effects must be accounted for when examining actinometry measurements of fluorine concentration. The material effects are believed to be due to the liberation of trapped oxygen in certain materials (such as alumina and quartz) which inhibit the recombination of fluorine.

\section{ACKNOWLEDGMENTS}

This work is sponsored by the Semiconductor Rcscarch Corporation under Contract No. 90-MC7-085. One of us (M.L.P.) wishes to acknowledge the support of an SRC Fellowship.
'J. W. Coburn and M. Chen, J. Appl. Phys. 51, 3134 (1980).

${ }^{2}$ R. d'Agostino, F. Cramarossa, S. DeBenedictis, and G. Ferraro, J. Appl. Phys. 52, 1259 (1981).

${ }^{3}$ J. W. Coburn and M. Chen, J. Vac. Sci. Technol. 18, 353 (1981).

${ }^{4}$ V. M. Donnelly, D. L. Flamm, W. C. Dautremont-Smith, and D. J. Werder, J. Appl. Phys. 55, 242 (1984)

${ }^{5}$ R. A. Gottscho and V. M. Donnelly, J. Appl. Phys. 56, 245 (1984).

${ }^{6}$ R. d'Agostino, F. Camarossa, S. DeBenedictis, F. Fracassi, L. Laska, and K. Masek, Plasma Chem. Plasma Process. 5, 239 (1985).

${ }^{7}$ A. D. Kichards, B. E. Thompson, K. D. Allen, and H. H. Sawin, J. Appl. Phys. 62, 792 (1987).

${ }^{8}$ P. Schoenborn and R. Patrick, J. Electrochem. Soc. 136, 199 (1989).

${ }^{9}$ I. C. Plumb and K. P. Ryan, Plasma Chem. Plasma Proc. 6, 205 (1986).

${ }^{10}$ D. Edelson and D. L. Flamm, J. Appl. Phys. 56, 1522 (1984).

"G. Smolinshky and D. L. Flamm, J. Appl. Phys. 50, 4982 (1979).

${ }^{12}$ I. C. Plumb and K. P. Ryan, Plasma Chem. Plasma Proc. 6, 11 (1986).

${ }^{13}$ D. L. Flamm, Solid State Technol. 21, 109 (1979). 